\title{
A Modified Fuzzy C-Means Clustering with Spatial Information for Image Segmentation
}

\author{
Hamed Shamsi and Hadi Seyedarabi, Member, IACSIT
}

\begin{abstract}
A traditional approach to segmentation of magnetic resonance (MR) images is the Fuzzy C-Means (FCM) clustering algorithm. However, the conventionally standard FCM algorithm is sensitive to noise. To overcome the above problem, a modified FCM algorithm (called MS-FCM later) for MRI brain image segmentation is presented in this paper. The algorithm is realized by incorporating the spatial neighborhood information into the standard FCM algorithm and modifying the membership weighting of each cluster. In The proposed algorithm every point of the data set has a weight in relation to every cluster. Therefore this weight permits to have a better classification especially in the case of noise data. The proposed algorithm is applied to both artificial synthesized image and real image. Segmentation results demonstrate that the presented algorithm performs more robust to noise than the standard FCM algorithm.
\end{abstract}

Index Terms-Fuzzy C-Means, spatial information, image segmentation, membership weighting.

\section{INTRODUCTION}

Segmentation of brain tissues in MRI (Magnetic Resonance Imaging) images plays a crucial role in three-dimensional volume visualization, quantitative morph metric analysis and structure-function mapping for both scientific and clinical investigations. Medical imaging provides effective and non-invasive mapping of the anatomy of subjects. Common medical imaging modalities include Xray, CT, ultrasound, and MRI. Medical imaging analysis is usually applied in one of two capacities: a) to gain scientific knowledge of diseases and their effect on anatomical structure in vivo, and b) as a component for diagnostics and treatment planning. MRI provides detailed images of tissues and is used for both human brain and body studies. Data obtained from MR images is used for detecting tissue deformities such as cancers and injuries [1]. It aims to partition an image into a set of non-overlapping regions whose union is the original image.

FCM clustering algorithm, an unsupervised clustering technique, has been successfully used for image segmentation [2], [3]. Compared with hard C-Means algorithm [4], FCM is able to preserve more information from the original image. Its advantages include a straightforward implementation, fairly robust behavior, applicability to multichannel data, and the ability to model

Manuscript received June 18, 2012; revised August 5, 2012.

H. Shamsi is with the Department of Electrical and Computer Engineering, University of Ataturk, Turkey (e-mail: Hamed.shamsi@atauni.edu.tr).

H. Seyedarabi is with the Department of Electrical and Computer Engineering, University of Tabriz, Tabriz, Iran (e-mail: Seyedarabi@tabrizu.ac.ir). uncertainty within the data. A major disadvantage of its use in imaging applications, however, is that FCM does not incorporate information about spatial context, causing it to be sensitive to noise and other imaging artifacts. The pixels on an image are highly correlated, i.e. the pixels in the immediate neighborhood possess nearly the same feature data. Therefore, the spatial relationship of neighboring pixels is an important characteristic that can be of great aid in imaging segmentation. The spatial function is the weighted summation of the membership function in the neighborhood of each pixel under consideration. However, the standard FCM does not take into account spatial information, which makes it very sensitive to noise. In a standard FCM technique, a noisy pixel is wrongly classified because of its abnormal feature data.

This paper introduces a modified segmentation algorithm for FCM clustering by incorporating spatial information and altering the membership weighting of each cluster with Fuzziness weighting exponent. The proposed algorithm greatly attenuates the effect of noise and biases the algorithm toward homogeneous clustering.

The organization of the paper is as follows. In Section 2, traditional fuzzy c-means algorithm and spatial fuzzy c-means are introduced. In Section 3, we obtain the fuzzy c-means cluster segmentation algorithm based on modified membership and modified cluster center. The experimental comparisons are presented in Section 4. Finally, in Section 5, we conclude and address the future work.

\section{FUZZY C-MEANS}

\section{A. Traditional Fuzzy C-Means}

The segmentation of imaging data involves partitioning the image space into different cluster regions with similar intensity image values. The most medical images always present overlapping gray-scale intensities for different tissues. Therefore, fuzzy clustering methods are particularly suitable for the segmentation of medical images. There are several FCM clustering applications in the MRI segmentation of the brain. The Fuzzy c-means (FCM) can be seen as the fuzzified version of the k-means algorithm. It is a method of clustering which allows one piece of data to belong to two or more clusters. This method (developed by Dunn [5] and Modified by Bezdek [6]) is frequently used in pattern recognition. The algorithm is an iterative clustering method that produces an optimal c partition by minimizing the weighted within group sum of squared error objective function JFCM: 


$$
J_{F C M}=\sum_{j=1}^{N} \sum_{i=1}^{c} u_{i j}{ }^{m}\left|x_{j}-v_{i}\right|^{2}
$$

where $X=\left\{x_{1}, x_{2}, \ldots, x_{n}\right\} \subseteq R^{p}$ is the data set in the p-dimensional vector space, $\mathrm{p}$ is the number of data items, $c$ is the number of clusters with $2 \leq c \leq n-1 . V=\left\{v_{1}, v_{2}, \ldots, v_{c}\right\}$ is the $c$ centers or prototypes of the clusters, vi is the p-dimension center of the cluster $i . U=\left\{\mu_{i j}\right\}$ represents a fuzzy partition matrix with $u_{i j}=u_{i}\left(x_{j}\right)$ is the degree of membership of $x_{j}$ in the ith cluster, $x_{j}$ is the jth of p-dimensional measured data. The fuzzy partition matrix satisfies:

$$
\begin{aligned}
& 0<\sum_{j=1}^{n} u_{i j}\langle n, \forall i \in\{1, \ldots \ldots, c\} \\
& \sum_{j=1}^{c} u_{i j}=1, \forall j \in\{1, \ldots \ldots, n\} \\
& 0 \leq U_{i j} \leq 1 \quad, \quad \forall i, j
\end{aligned}
$$

The parameter $m$ is a weighting exponent on each fuzzy membership and determines the amount of fuzziness of the resulting classification; it is a fixed number greater than one. The objective function JFCM can be minimized under the Constraint of U. specifically, taking of JFCM with respect to uij and vi and zeroing then respectively, tow necessary but not sufficient conditions for JFCM to be at its local extreme will be as the following:

$$
\begin{aligned}
& U_{i j}=\sum_{k=1}^{c}\left(\frac{\left\|x_{j}-v_{i}\right\|}{\left\|x_{j}-v_{k}\right\|}\right)^{\frac{-2}{m-1}} \\
& \text { for } i=1,2, \ldots . . c \quad \text { and } \quad \text { for } j=1,2, \ldots \ldots, n \\
& V_{i}=\frac{\sum_{j=1}^{n}\left(u_{i j}\right)^{m} x_{j}}{\sum_{j=1}^{n}\left(u_{i j}\right)^{m}} \quad \text { for } i=1,2, \ldots \ldots . c
\end{aligned}
$$

Although FCM is a very useful clustering method, its memberships do not always correspond well to the degree of belonging of the data, and may be inaccurate in a noisy environment, because the real data unavoidably involves some noises.

\section{B. Spatial Fuzzy C-Means (SFCM)}

One of the important characteristics of an image is that neighboring pixels have similar feature values, and the probability that they belong to the same cluster is great. The spatial information is important in clustering, but it is not utilized in a standard FCM algorithm [7]. To exploit the spatial information, a spatial function is defined as:

$$
S_{i j}=\sum_{k \in N B\left(x_{j}\right)} U_{i k}
$$

The spatial function is the weighted summation of the membership function in the neighborhood of each pixel under consideration. Just like the membership function, the spatial function sij represents the probability that pixel $x j$ belongs to ith clustering. The spatial function is the largest if all of its neighborhood pixels belong to ith clustering, and is the smallest if none of its neighborhood pixels belong to ith clustering. The spatial function is incorporated into membership function as follows:

$$
\begin{aligned}
& U_{i j}^{*}=\frac{U_{i j}^{p} * S_{i j}^{q}}{\sum_{k=1}^{c} U_{k j}^{p} * S_{k j}^{q}} \\
& \text { for } i=1,2, \ldots ., \text { and } j=1,2, \ldots \ldots, n
\end{aligned}
$$

where $\mathrm{p}$ and $\mathrm{q}$ are parameters to control the relative importance of both functions. In a homogenous region, the spatial functions simply fortify the original membership, and the clustering result remains unchanged. However, for a noisy pixel, this formula reduces the weighting of a noisy cluster by the labels of its neighboring pixels. As a result, misclassified pixels from noisy regions or spurious blobs can easily be corrected.

There are two steps at each clustering iteration. The first step is to calculate the membership function in the spectral domain and the second step is to map the membership information of each pixel to the spatial domain and then compute the spatial function from that.

\section{A Modified FuZZY C-MeAns Clustering With SPATIAL INFORMATION}

We can compare the membership of central pixel with the one of neighbor pixels in a window to analysis whether the central pixel is classified rightly or not. This spatial relationship is important in clustering; therefore a new spatial function is defined as:

$$
S_{i j}{ }^{*}=\sum_{k \in H\left(x_{j}\right)} U_{i k} \beta_{k 1}+\frac{\sum_{k \in H\left(x_{j}\right)} U_{i k} \beta_{k 2}}{\sum_{t=1}^{c} \sum_{k \in H\left(x_{j}\right)} U_{t k}}
$$

where $\mathrm{H}(\mathrm{xj})$ represents a square window centered on pixel $\mathrm{xj}$ in the spatial domain. Introduced new spatial function has two parts. The first part is controlled by $\beta_{k 1}$ coefficient caused that misclassified pixels from noisy regions can be easily corrected. The second part is controlled by $\beta_{k 2}$ coefficient caused membership function quantitative according to distance between pixels.

$$
\begin{aligned}
& \beta_{k 1}=\frac{1}{1+\exp \left(\theta_{1}\|j-k\|\right)} \\
& \beta_{k 2}=\frac{1}{1+\exp \left(\theta_{2}\left\|x_{j}-x_{k}\right\|\right)}
\end{aligned}
$$

The spatial function is incorporated into membership function as follows: 


$$
\begin{aligned}
& U_{i j}^{*}=\frac{U_{i j}^{p} * s_{i j}^{* q}}{\sum_{k=1}^{c} U_{k j}^{p} * s_{i j}^{* q}} \\
& \text { for } i=1,2, \ldots ., c \text { and } j=1,2, \ldots ., n
\end{aligned}
$$

The choice of an appropriate objective function is the key to the success of the cluster analysis and to obtain better quality clustering results; so the clustering optimization is based on objective function [8]. To meet a suitable objective function, we started from the following set of requirements: The distance between clusters and the data points assigned to them should be minimized and the distance between clusters should to be maximized [9]. The attraction between data and clusters is modeled by term (12); it is the formula of the objective function. Wen-Liang Hung proposed a new algorithm called Modified Suppressed Fuzzy c-means (MS-FCM), which significantly ameliorates the performance of FCM due to a prototype-driven learning of parameter $\alpha$ [10]. The learning process of $\alpha$ is based on an exponential separation strength between clusters and is updated at each iteration. The formula of this parameter is:

$$
\alpha=\exp \left(-\min \frac{\left\|v_{i}-v_{k}\right\|^{2}}{\beta}\right)
$$

where $\beta$ is a normalized term so that we choose $\beta$ as a sample variance. That is, we define $\beta$ :

$$
\beta=\frac{\sum_{j=1}^{n}\left\|x_{j}-\bar{x}\right\|^{2}}{n} \quad \text { where } \bar{x}=\frac{\sum_{j=1}^{n} x_{j}}{n}
$$

But the remark which must be mentioned here is the common value used for this parameter by all the data at each iteration, which may induce in error. We propose a new parameter which suppresses this common value of $\alpha$ and replaces it by a new parameter like a weight to each vector. Or every point of the data set has a weight in relation to every cluster. Therefore this weight permits to have a better classification especially in the case of noise data. So the weight is calculated as follows:

$$
W_{j i}=\frac{1}{1+\exp \left(-\frac{\left\|x_{j}-v_{i}\right\|^{2}}{\sum_{j=1}^{n}\left\|x_{j}-\bar{v}\right\|^{2} * c / n}\right)}
$$

where $w_{j i}$ is weight of the point $\mathrm{j}$ in relation to the class $i$. This weight is used to modify the fuzzy and typical partition.

The objective function of the MS-FCM can be formulated as follows:

$$
J_{m s f c m}=\sum_{k=1}^{n} \sum_{i=1}^{c}\left(u_{i k}^{m} W_{j i}^{m}\right)\left|x_{k}-v_{i}\right|^{2}
$$

Modified FCM approach is given below:

- Step 1: Select the data set.
- Step 2: Fix $\mathrm{m}>1$ and $2 \leq c \leq n-1$ and give $\mathrm{c}$ initial cluster centers $V_{i}$.

- Step 3: Compute $\mathrm{U}_{\mathrm{ij}}$ with $V_{i}$ by Eq. (3).

- Step 4: Compute $\beta_{k 1}$ and $\beta_{k 2}$ by Eq. (8) and (9).

- Step 5: Compute $S^{*}{ }_{i j}$ and $W_{j i}$ by Eq. (7) and (13).

- Step 6: Update the membership matrices by Eq. (10), Update the centroids using (4).

- Step 7: if $\left\|V_{\text {new }}-V_{\text {old }}\right\| \leq \varepsilon$ Stop the iteration otherwise, go to step 4.

\section{EXPERIMENTAL RESUlTS}

In order to verify the effectiveness of the proposed algorithm, we give some experiments using both artificial synthesized image and real image to compare the performance of the proposed algorithm with that of the standard FCM algorithm.

\section{A. Experimental Results on Synthetic Image}

Fig. 1a shows a synthesized image with three classes, the image degraded by the Salt-Pepper noise (noisy density $d=$ $0.02)$ and Gaussian noise $(u=0, \delta=0.02)$ is shown in Fig. $1 \mathrm{~b}$ and $1 \mathrm{c}$, respectively.

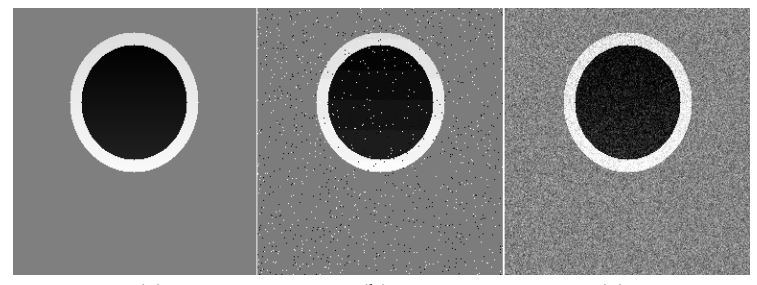

(a)

(b)

(c)

Fig. 1. Synthesized image: (a) original image, (b) image degraded by Salt-Pepper noise, (c) image degraded by Gaussian noise.

Figs. 2a-2b-2c display the clustering results of the Salt-Pepper degraded image using the FCM, SFCM and MS-FCM algorithm, correspondingly, the clustering results on Gaussian degraded image were shown in Figs. 3a-3b-3c.

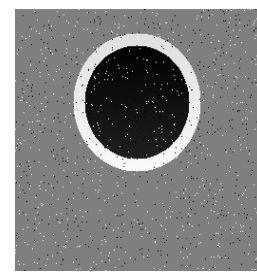

(a)

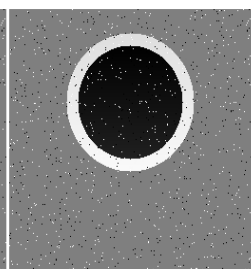

(b)

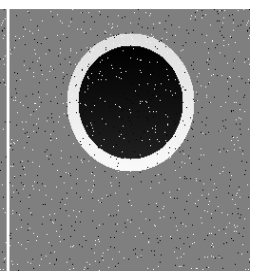

(c)
Fig. 2. Comparison of segmentation results on synthetic image which is corrupted by $2 \%$ salt- pepper noise. (a) FCM result, (b) SFCM result (c) MS-FCM result.

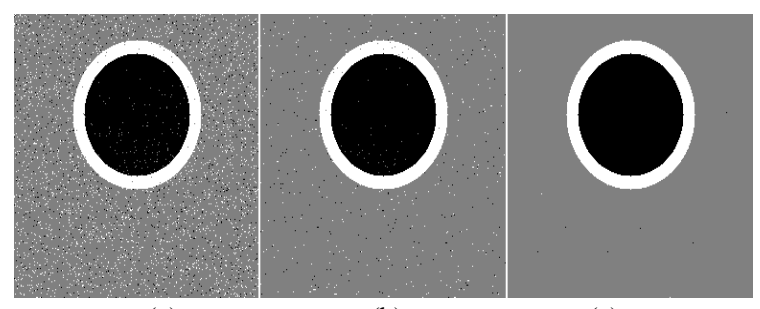

(a)

(b)

(c)

Fig. 3. Comparison of segmentation results on synthetic image which is corrupted by Gaussian noise $(u=0, \delta=0.02$ ) (a) FCM result (b) SFCM result (c) MS-FCM result. 
In order to obtain a quantitative comparison, two types of cluster validity functions, fuzzy partition and feature structure, are often used to evaluate the performance of clustering in different clustering methods. The representative functions for the fuzzy partition are partition coefficient $\mathrm{Vpc}$ [11] and partition entropy Vpe [12]. They are defined as follows:

$$
\begin{gathered}
\mathrm{V}_{\mathrm{pc}}(U)=\frac{1}{n}\left(\sum_{K=1}^{n} \sum_{i=1}^{c} u_{i k}^{2}\right) \\
\mathrm{V}_{\mathrm{pe}}(U)=-\frac{1}{n}\left(\sum_{K=1}^{n} \sum_{i=1}^{c} u_{i k} \log u_{i k}\right)
\end{gathered}
$$

The idea of these validity functions is that the partition with fuzziness means better performance. As a result, the best clustering is achieved when the value $\mathrm{Vpc}$ is maximal or $\mathrm{Vpe}$ is minimal.

Table I tabulates the Vpc and Vpe and number of iteration of the three algorithms on two different noise degraded images shown in Figs. $1 \mathrm{~b}$ and 1c, respectively.

TABLE I: COMPRESSION OF THE CLUSTERING RESULTS ON TWO KIND NOISE DEGRADED SYNTHETIC IMAGE USING THREE FCM ALGORITHMS

\begin{tabular}{cllll}
\hline \hline Noise type & Algorithm & Vpc & Vpe & iter \\
\hline Salt-pepper & FCM & 0.9961 & 0.0049 & 12 \\
Salt-pepper & SFCM & 0.9984 & 0.0016 & 6 \\
Salt-pepper & MS-FCM & 0.9991 & 0.0008 & 5 \\
Gaussian & FCM & 0.8308 & 0.1386 & 43 \\
Gaussian & SFCM & 0.9630 & 0.0323 & 11 \\
Gaussian & MS-FCM & 0.9964 & 0.0042 & 6 \\
\hline \hline
\end{tabular}

From Table I, obviously, MS-FCM achieves better performance than FCM and SFCM, which demonstrates the modified SFCM algorithm (MS-FCM) a visually significant improvement of robustness to noise over the FCM and SFCM algorithm.

\section{B. Experimental Result on Real Image}

Fig.4a shows an MRI brain image with $256 * 256$ pixels. The image degraded by the Gaussian noise $(u=0, \delta=0.02)$ and the Salt-Pepper noise with noisy density $d=0.02$ is shown in Fig. $4 \mathrm{~b}$ and $4 \mathrm{c}$, respectively.

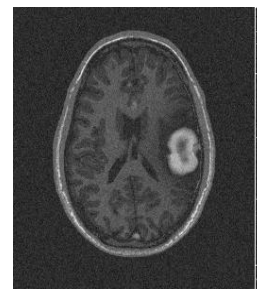

(a)

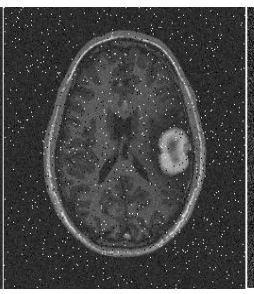

(b)

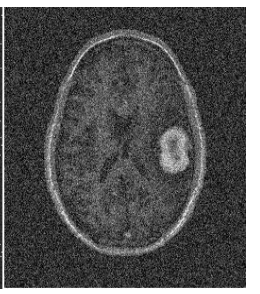

(c)
Fig. 4. MRI image: (a) original image, (b) image degraded by Salt-Pepper.

Figs. 5a-5b-5c display the clustering results of the Salt-Pepper degraded image using the FCM and SFCM and MS-FCM algorithm, correspondingly, the clustering results on Gaussian degraded image were shown in Figs. 6a-6b-6c.

Visually, the comparison of segmentation results indicates that MS-FCM algorithm greatly reduces the effect of noise.

Table II summarizes the partition coefficient Vpc and the partition entropy Vpe of the two algorithms on the two different degraded images.

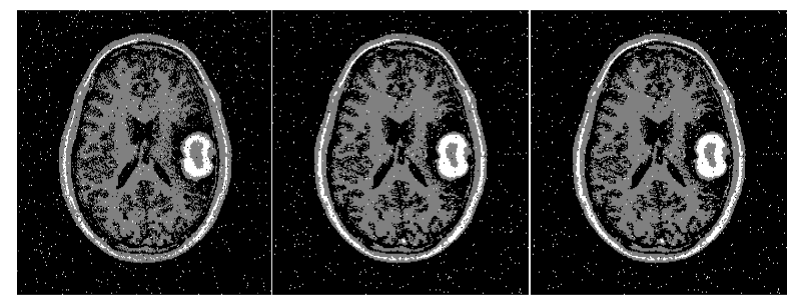

(a)

(b)

(c)

Fig. 5. Comparison of segmentation results on MRI image which is corrupted by $2 \%$ Salt- Pepper noise. (a) FCM result (b) SFCM result (c) MS-FCM result.

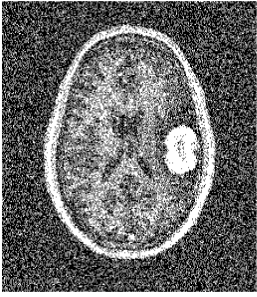

(a)

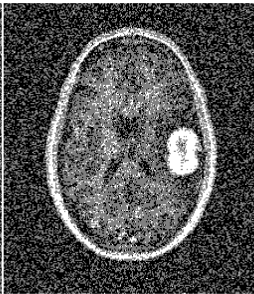

(b)

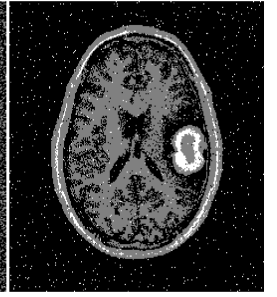

(c)
Fig. 6. Comparison of segmentation results on MRI image which is corrupted by Gaussian noise $(u=0, \delta=0.02)$ (a) FCM result (b) SFCM result (c) MS-FCM result.

TABLE II: COMPRESSION OF THE CLUSTERING RESULTS ON TWO KIND NOISE DEGRADED MRI IMAGE USING THREE FCM ALGORITHMS.

\begin{tabular}{lllll}
\hline \hline Noise type & Algorithm & Vpc & Vpe & iter \\
\hline Salt-pepper & FCM & 0.8631 & 0.1081 & 81 \\
Salt-pepper & SFCM & 0.8647 & 0.1066 & 79 \\
Salt-pepper & MS-FCM & 0.9130 & 0.0628 & 52 \\
Gaussian & FCM & 0.7669 & 0.1812 & 77 \\
Gaussian & SFCM & 0.7901 & 0.1573 & 71 \\
Gaussian & MS-FCM & 0.8359 & 0.1203 & 42 \\
\hline \hline
\end{tabular}

Table II shows that our proposed algorithm improves significantly the performances of clustering both Gaussian degraded image and Salt-Pepper degraded image compare to the standard FCM algorithm.

\section{CONCLUSION}

The main drawback of the standard FCM for image segmentation is that the objective function does not take into consideration the spatial information in the image, but deal with images as the same as separate points. Therefore, as mentioned in many literatures $[13,14]$ the standard FCM algorithm is sensitive to noise and a noisy pixel is always wrongly classified because of its abnormal feature.

In this paper, we proposed a new modify spatial FCM that incorporates the spatial information into the membership function to improve the segmentation results. In the new spatial function we used two contribution factors. The first one was according to distances between central pixels with neighbor pixels. The second factor was calculated according to value difference of central pixel with neighbor pixels. Using of these contribution factors caused that spatial function is made of according to distance and value pixels.

The new method was tested on MRI images and evaluated by using various cluster validity functions. Preliminary results showed that the effect of noise in segmentation was considerably less with the new algorithm than with the conventional FCM. 


\section{REFERENCES}

[1] B. Buyuksarac and M. Ozkan, "Image segmentation in mri using true ti and true Pd values," In Engineering in Medicine and Biology Society, 2001. Proceedings of the 23rd Annual International Conference of the IEEE, vol. 3, pp. $2661-2664,2001$.

[2] D. L. Pham and J. L. Prince, "An adaptive fuzzy c-means algorithm for image segmentation in the presence of intensity in homogeneities," Pattern Recognition. Lett. vol. 20, pp 57-68, 1999.

[3] W. J. Chen, M. L. Giger, and U. Bick, "A fuzzy c-means (FCM)-based approach for computerized segmentation of breast lesions in dynamic contrast enhanced MRI images," Acad. Radiol, vol. 13, pp. 63-72, 2006

[4] J. M. Gorriz, J. Ramirez, E. W. Lang, and C. G. Puntonet, "Hard c-means clustering for voice activity detection," Speech Commun, vol. 48, pp. 1638-1649, 2006.

[5] J. C. Dunn, "A fuzzy relative of the ISODATA process and its use in detecting compact well-separated clusters," J. Cybernetics, vol. 3, no. 3, pp. 32-57, 1973.

[6] J. C. Bezdek, "Pattern recognition with fuzzy objective function algorithms," New York, Plenum, 1981.

[7] K.-S. Chuang, H.-L. Tzeng, S. Chen, J. Wu, and T.-J. Chen , "Fuzzy c-means clustering with spatial information for image segmentation," Computerized Medical Imaging and Graphics vol. 30, pp. 9-15, 2006.

[8] Y. Zhonghang, T. Yangang, S. Funchun, and S. Zengqi, "Fuzzy clustering with novel serable criterion," Tsinghua Science and Technology, vol. 11, no. 1, pp. 50-53, February 2006.

[9] H. Timm, C. Borgelt, C. Doring, and R. Kruse, "An extension to possibilistic fuzzy cluster analysis," Fuzzy Sets and Systems, vol. 147, pp. 3-16, 2004.

[10] W. L. Hung, M. Yang, and D. Chen, "Parameter selection for suppressed fuzzy c-means with an application to MRI segmentation," Pattern Recognition Letters, 2005.

[11] J. C. Bezdek, "Cluster validity with fuzzy sets," Journal of Cybernetics, vol. 3, 1974, pp. 58-73.
[12] J. C. Bezdek, "Mathematical models for systematic and taxonomy," In: Proceedings of Eight International Conference on Numerical Taxonomy, July. 2004, pp. 143-166.

[13] W. J. Chen, M. L. Giger, and U. Bick, "A fuzzy c means (FCM)-based approach for computerized segmentation of breast lesions in dynamic contrast enhanced MRI images," Acad. Radiol, vol. 13, pp. 63-72, 2006.

[14] M. Y. Siyal and L. Yu, "An intelligent modified fuzzy c-means based algorithm for bias estimation and segmentation of brain MRI," Pattern Recognition Letter, vol. 26, pp. 2052-2062, 2005.

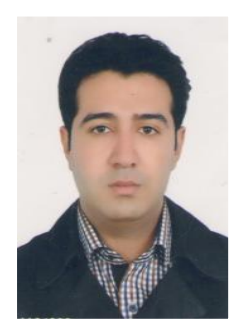

Hamed Shamsi received the B.Sc. degrees from Islamic Azad University of Urumiyeh in 2008 and the M.Sc. degrees from Islamic Azad University of Najafabad Branch in 2010 all in Electrical Engineering field (telecommunication). $\mathrm{He}$ is currently Ph.D. student in Ataturk University, turkey. His research interests are image processing, image segmentation, medical signal processing, pattern recognition, and fuzzy logic.

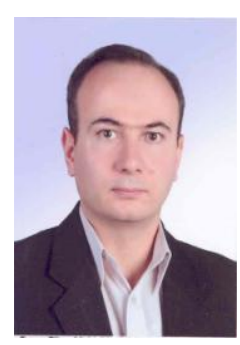

Hadi Seyedarabi Received B.S. degree from University of Tabriz, Iran, in 1993, the M.S. degree from K.N.T. University of technology, Tehran, Iran in 1996 and Ph.D. degree from University of Tabriz, Iran, in 2006 all in Electrical Engineering. He is currently an assistant professor of Faculty of Electrical and Computer Engineering in University of Tabriz, Tabriz, Iran. His research interests are image processing, computer vision, Human-Computer Interaction, facial expression recognition and facial animation. 